\title{
Successful Treatment of Primary Sclerosing Cholangitis with a Steroid and a Probiotic
}

\author{
Masaki Shimizu Hidenori Iwasaki Shintaro Mase \\ Akihiro Yachie \\ Department of Pediatrics, School of Medicine, Institute of Medical, \\ Pharmaceutical and Health Sciences, Kanazawa University, Kanazawa, Japan
}

\section{Key Words}

Primary sclerosing cholangitis $\cdot$ Inflammatory bowel disease $\cdot$ Probiotics

\begin{abstract}
Primary sclerosing cholangitis (PSC) is a serious disease that not only affects quality of life but can also have a significant effect on patient survival. The treatment for PSC is primarily supportive with the aim of controlling cholestatic symptoms and preventing complications. Ursodeoxycholic acid may induce biochemical improvements in affected patients; however, long-term pediatric studies to determine its possible benefits in young patients are lacking. Thus, the treatment of pediatric PSC remains a significant clinical challenge. We describe a patient with PSC and undetermined colitis who was treated with a combination of a steroid, salazosulfapyridine, and a probiotic. This treatment provided benefits both for PSC and the undetermined colitis. These findings suggest that bacterial flora and gut inflammation are closely associated with the pathogenesis of inflammatory bowel disease-related PSC. Suppression of bowel inflammation and maintenance of bacterial homeostasis may be important for treating PSC.
\end{abstract}

\section{Introduction}

Primary sclerosing cholangitis (PSC) is a chronic cholestatic liver disease characterized by inflammation and progressive obliterative fibrosis of the intrahepatic and/or extrahepatic bile ducts. There is a close association between PSC and inflammatory bowel disease (IBD) [1]. However, the pathogenesis of IBD-related PSC remains unknown. Increasing evidence suggests that immune mechanisms play a critical role in the pathogenesis of PSC [2], and it has been hypothesized that gut-homing lymphocytes may be the common denominator underlying both IBD and PSC [2]. 
A possible nonimmune mechanism involved in PSC pathogenesis is the release of toxic agents either by gut bacteria or following ischemic injury [3]. Interestingly, antibiotics have been used to treat PSC in several patient series over the years, and they exhibited benefits in some patients $[3,4]$. These observations suggest that bacterial flora and gut inflammation might be closely associated with the pathogenesis of both IBD and PSC.

PSC is a serious disease that not only affects quality of life but can also have a significant effect on patient survival. The treatment for PSC is primarily supportive with the aim of controlling cholestatic symptoms and preventing complications. Ursodeoxycholic acid (UDCA) may induce biochemical improvements in patients. However, long-term pediatric studies to determine its possible benefits in young patients are lacking. Thus, treating pediatric PSC remains a significant clinical challenge.

In this report, we describe a patient with PSC and undetermined colitis who was successfully treated with a combination of a steroid, salazosulfapyridine, and a probiotic.

\section{Case Report}

A 13-year-old boy with a 4-week history of fever, abdominal pain and associated anemia and liver dysfunction was referred to us. His laboratory data on admission showed the following: aspartate aminotransferase $80 \mathrm{IU} / \mathrm{l}$, alanine aminotransferase $102 \mathrm{IU} / \mathrm{l}$, alkaline phosphatase 2,663 IU/l, $\gamma$-glutamyl transpeptidase $524 \mathrm{IU} / \mathrm{l}$, immunoglobulin G 2,180 mg/dl, and erythrocyte sedimentation rate $111 \mathrm{~mm} / \mathrm{h}$ (table 1). Colonoscopy revealed pancolitis including erosive lesions with rectal sparing, but without granulomatous changes. A diagnosis of undetermined colitis was made.

Magnetic resonance cholangiopancreatography showed an irregular contour of the intrahepatic bile duct (fig. 1a). A liver biopsy revealed severe biliary tract outflow obstruction and chronic active inflammation involving the biliary ducts (fig. 1b, c). Findings of hepatocellular involvement consistent with autoimmune hepatitis were not significant. Endoscopic retrograde cholangiography showed narrowing and irregularities of the intrahepatic bile ducts. A diagnosis of PSC associated with undetermined colitis was made.

Treatment with UDCA (600 mg/day) and mesalazine $(2,000 \mathrm{mg} /$ day $)$ was started, after which his symptoms improved temporarily. However, he had fever and bloody diarrhea 2 weeks after initiating this treatment. Mesalazine was replaced with prednisolone $(30 \mathrm{mg} /$ day $)$, salazosulfapyridine $(3,000 \mathrm{mg} /$ day) and a probiotic (Lactobacillus casei Shirota, $3 \mathrm{~g} /$ day). Two weeks later, the patient's symptoms and laboratory results had improved (table 1).

The prednisolone dose was gradually tapered off. A repeat biopsy at 30 months showed significant improvements in inflammatory cell infiltration and periductal fibrosis and a decrease in fibrotic areas from 10.5 to $3.6 \%$ in each specimen (fig. 1d). Three years later, the patient continues to take UDCA, salazosulfapyridine and probiotics. He is in complete remission.

\section{Discussion}

Immunosuppressive therapy with corticosteroids and/or azathioprine is often used in the treatment of IBD. These drugs have also been used for treating pediatric patients with PSC [5]. Children with autoimmune cholangitis should be expected to respond to immunosuppressive therapy when liver histology shows interface hepatitis, immunoglobulin G levels are elevated and autoimmune markers are present. 
Antibiotics have been used to treat PSC and exhibited benefits in some cases [3, 4]. These findings suggest that bacterial flora and gut inflammation may be closely associated with the pathogenesis of both IBD and PSC.

Probiotics are nonpathogenic viable microorganisms that have beneficial effects for host health, disease prevention and/or treatment. Clinical trials have shown some beneficial effects of probiotics in IBD [6]. The suggested mechanisms for probiotic regulation in IBD therapy are the following. First, probiotics block bacterial pathogenic effects by producing bactericidal substances and competing with pathogens and toxins for adherence to the intestinal epithelium. Second, probiotics regulate immune responses by enhancing innate immunity and modulating pathogen-induced inflammation via toll-like receptor-regulated signaling pathways. Third, probiotics regulate intestinal epithelial homeostasis by promoting intestinal epithelial cell survival, enhancing barrier function and stimulating protective responses.

For our patient, the combination of immunosuppressive therapy and a probiotic provided benefits for both IBD and PSC, which suggests that bacterial flora and gut inflammation are closely associated with the pathogenesis of IBD-related PSC.

Suppressing bowel inflammation and maintaining bacterial homeostasis in the gut is important in the treatment of PSC. The results of a randomized controlled trial showed that probiotics alone could not control symptoms or improve liver function in adult PSC patients [7]. However, additional studies are needed to determine the effects of probiotics as an adjunctive therapy in pediatric IBD-related PSC.

\section{Acknowledgements}

The authors express their gratitude to the parents of the patient, who graciously authorized the publication of the information presented here.

\section{Disclosure Statement}

The authors declare no conflicts of interest.

Table 1. Laboratory data on admission

\begin{tabular}{llll}
\hline & Onset & $\begin{array}{l}\text { Two weeks } \\
\text { after treatment }\end{array}$ & Normal \\
\hline $\begin{array}{lll}\text { Aspartate aminotransferase, IU/l } \\
\text { Alanine aminotransferase, IU/l }\end{array}$ & 80 & 14 & $13-33$ \\
Alkaline phosphatase, IU/l & 102 & 13 & $6-27$ \\
Gamma-glutamyl transpeptidase, IU/l & 2,663 & 372 & $115-319$ \\
Immunoglobulin G, mg/dl & 524 & 16 & $10-47$ \\
Erythrocyte sedimentation rate, $\mathrm{mm} / \mathrm{h}$ & 2,180 & 1,570 & $870-1,700$ \\
\hline
\end{tabular}




\begin{tabular}{r|l|l|l} 
Case Reports in & $\begin{array}{l}\text { Case Rep Gastroenterol 2012;6:249-253 } \\
\text { DOI: 10.1159/000338834 }\end{array}$ & $\begin{array}{l}\text { Published online: } \\
\text { May 8, 2012 }\end{array}$ & $\begin{array}{l}\text { @ 2012 S. Karger AG, Basel } \\
\text { ISSN 1662-0631 } \\
\text { www.karger.com/crg }\end{array}$ \\
\hline
\end{tabular}
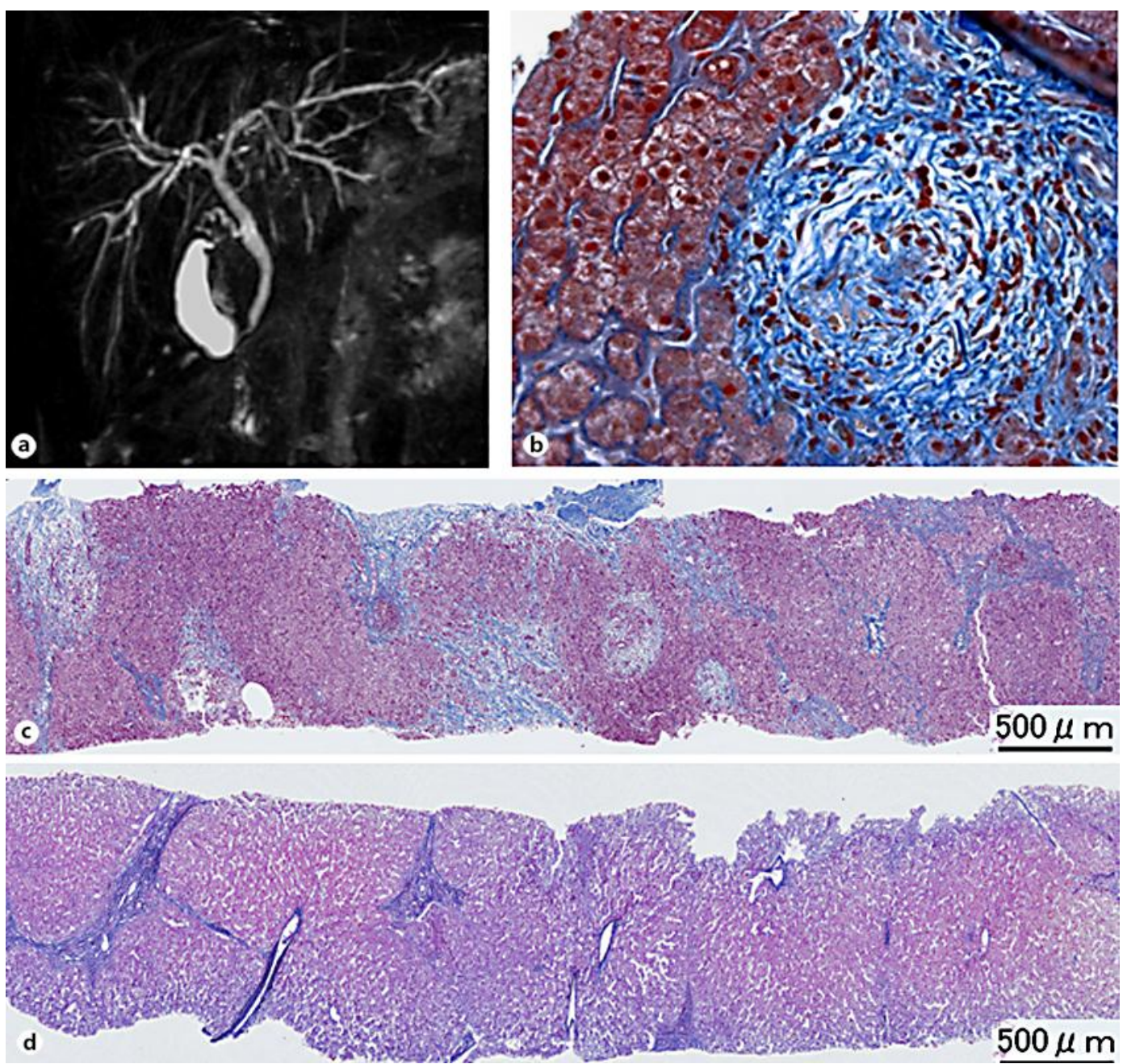

Fig. 1. Pathological findings in PSC. a Magnetic resonance cholangiopancreatography showed an irregular contour of the intrahepatic bile duct. b, $\mathbf{c}$ The first liver biopsy revealed severe changes, including biliary tract outflow obstruction and chronic active inflammation involving the biliary tree (Azan staining; original magnification: $\mathrm{b} \times 400, \mathrm{c} \times 100$ ). $\mathrm{d}$ The second biopsy showed significant improvements in inflammatory cell infiltration and periductal fibrosis. Fibrotic areas had decreased from 10.5 to $3.6 \%$ in each specimen (Azan staining; original magnification: $\times 100$ ).

\section{References}

1 Faubion WA, Loftus EV, Sandborn WJ, Freese DK, Perrault J: Pediatric 'PSC-IBD': a descriptive report of associated inflammatory bowel disease among pediatric patients with PSC. J Pediatr Gastroenterol Nutr 2001;33:296-300.

-2 Grant AJ, Lalor PF, Hubscher SG, Briskin M, Adams DH: MAdCAM-1 expressed in chronic inflammatory liver disease supports mucosal lymphocyte adhesion to hepatic endothelium (MAdCAM-1 in chronic inflammatory liver disease). Hepatology 2001;33:1065-1072.

- 3 Davies YK, Cox KM, Abdullah BA, Safta A, Terry AB, Cox KL: Long-term treatment of primary sclerosing cholangitis in children with oral vancomycin: an immunomodulating antibiotic. J Pediatr Gastroenterol Nutr 2008;47:61-67.

4 Elfaki DA, Lindor KD: Antibiotics for the treatment of primary sclerosing cholangitis. Am J Ther 2011;18: 261-265. 
-5 Ibrahim SH, Lindor KD: Current management of primary sclerosing cholangitis in pediatric patients. Pediatr Drugs 2011;13:87-95.

6 Isaacs K, Herfarth H: Role of probiotic therapy in IBD. Inflamm Bowel Dis 2008;14:1597-1605.

7 Vleggaar FP, Monkelbaan JF, van Erpecum KJ: Probiotics in primary sclerosing cholangitis: a randomized placebo-controlled crossover pilot study. Eur J Gastroenterol Hepatol 2008;20:688-692. 